\title{
Liderazgo y Gestión de la Calidad ¿Desafío o Destino?
}

\author{
M. Angustias Martín Quirós \\ Universidad de Granada \\ José Medina \\ Ray \& Berndtson
}

RESUMEN

La Calidad se ba asociado durante años a un conjunto de elementos tangibles de la gestión. No obstante, aunque las dimensiones tangibles sean componentes necesarios, no son suficientes para alcanzarla. Desde el propio objetivo que persigue: la satisfaccion de las personas implicadas, y como consecuencia. los medios que requiere -moticacion. implicación. compromiso personal- constituyen sus componentes menos visible pero los auténticamente diferenciadores. En este articulo se reflexiona sobre las caracteristicas, retos y' aspectos itiales para aquellos lideres cuya meta compartida es generar las condiciones para que pueda desarrollarse la Calidad con su gestión.

Palabras clave: Gestiōn de la Calidad Total: Liderazgo: Satisfacción: Implicación.

\section{ABSTRACT}

Quality has been largely associated to a group of management tangihle elements. Netertheless, although tangibles are necessary components, they are not enough to reach it. From the pursued objective: the people satisfaction, to the required means -motivation, implication, personal commitment- they constitute the less visible components but still the ones that makes the difference. In this paper it is presented on the characteristics. challenges and vital aspects for those leaders whose shared goal is to generate the conditions to develop Quality Management.

Key: words: Total Quality Management; Leadership; Satisfaction; Implication. 


\section{INTRODLCCIÓN}

Cuando se habla de calidad es frecuente asociarla con términos tales coms estándares, recursos, operaciones, procesos, control, costes, planificación o verificación. No obstante, cada vez con más entusiasmo se resalta la importancia del liderazgo y del lado humano de la calidad, materializada a través de la polítiç de recursos humanos y de su dirección como dimensiones críticas de la Gestión de la Calidad Total (GCT). No en vano calidad es generar satisfacción, una experiencia gratificante para las personas en contacto con el producto o servicio ? para generar satisfacción deben participar todas aquellas que, desde diferenter posiciones, contribuyen a generarlo y ofrecerlo.

Para satisfacer a sus clientes, las empresas deben arbitrar numerosas medidar tendentes a planificar, diseñar y reproducir sistemáticamente todas aquellas accionei que le lleven a ofrecer resultados homogéneos. Con ello reducen la variabilidad incorporan herramientas que posibilitan un seguimiento de las desviaciones que st van produciendo en cada paso donde se incorpora valor al proceso. En este sentide, la literatura es abundante y las aplicaciones realizadas en las empresas son cada ver más numerosas. Buen ejemplo de ello es la proliferación de compañías con certificados de organismos acreditados garantizando que han implantado sistemas de asegu. ramiento de la calidad de acuerdo con las Normas Internacionales ISO 9000. Sin embargo, la generación de satisfacción requiere sentir que se cumplen las expectativas, lo que va más allá de lo estrictamente objetivo y programable. Representa èl paso de gestionar unos recursos técnicos y materiales, que dentro de sus dificultades, resulta más sencillo, a generar ilusiones, entusiasmo y compromiso, a gestionar necesidades, voluntades, competencias y valores donde se desvanece la posibilidail de estandarización. Se pasa al mundo de lo subjetivo, donde el perfil personal y humano de los agentes, reclaman el protagonismo que les corresponde. Es un proceso donde el directivo tiene que poner en juego no sólo conocimientos sino también sus valores. De este complicado arte, al que miles de personas adscriben diariamente su tiempo, nos ocupamos en nuestro artículo.

\section{Generar Compromiso: El Desafí Permanente}

La calidad está inseparablemente vinculada a la generación de satisfacción y éstạ. a su vez, se ha convertido en indispensable para mantener la salud del negocio. Pari conseguir un crecimiento rentable y sostenido se precisa fidelizar a los clientes; para ello es preciso conseguir que éstos estén completamente satisfechos. En êl sector de la automoción, un cliente completamente satisfecho está 6 veces má predispuesto a volver a comprar el producto, mientras que un cliente insatisfechi transmite hasta a 11 potenciales clientes información negativa de la marca (Andersen, 1999 pág 39). 
Pero la satisfacción más que como un resultado debe perseguirse de forma constante en la gestión, propagándose en todos los eslabones de la cadena: los que se construyen a partir de ese proceso ininterrumpido que se forma entre cliente y proveedor interno, en el que cada cual asume, en turno, uno u otro rol así como con el exterior. Johannsson (1995) establece la relación directa entre la satisfacción de los miembros de la empresa y la del cliente en su descripción sobre las fortalezas de ABB Atoms que la hacen ser una candidata excelente para ganar el premio Sueco a la calidad.

La tarea preventiva que lleva a los resultados esperados consiste en proporcionar, cada cual en su papel de proveedor, todos los inputs de calidad. Se trata de una cadena que sobrepasa los límites de la empresa como lo muestra el modelo de partnerships de Siemens, en dónde la relación cliente-proveedor les lleva a compartir conocimientos con su cliente, estableciendo conjuntamente los mecanismos necesarios para optimizar tiempos y costes y para reducir drásticamente errores y contribuir así al éxito de los clientes de la organización.

Pero para que los inputs sean de calidad deben ofrecer, no sólo los tangibles significativos esperados por el receptor en el tiempo, cantidad y especificaciones, sino que también deben ir acompañados por elementos menos materiales, que posibiliten, por ejemplo, un clima positivo y de confianza. De esta forma. la gestión orientada a la satisfacción pone sus cimientos en las personas, cuenta con todas aquellas personas que desarrollan el proceso pero que paralelamente se desarrollan a sí mismos. Representa la diferencia entre realizar una operación rutinaria desprovista de sentido para quien la ejecuta o poner el alma, implicándose en la tarea. Significa buscar el compromiso y la implicación para la mejora porque son los seres humanos que integran una empresa quienes construyen o pueden contribuir a la destrucción de la misma. "...las empresas que crean más valor para el accionista son precisamente aquellas que mejor sirven a sus clientes externos, y esto lo logran a través del talento de sus profesionales, personas competentes y motivadas" (Arraiz et al. 1999 pág. 22)

Aunque el compromiso es una cualidad personal e intrasferible, crear las condiciones para la motivación, implicación y el propio compromiso es misión de la dirección. De hecho, el papel de la dirección se presenta en la literatura de GCT como una de sus dimensiones críticas. Los grandes modelos de la calidad, los que rigen los premios Deming en Japón, el Malcolm Braldrige en EE UU o el Europeo asignan un peso significativo a este criterio. El Modelo del Premio Europeo a la Calidad otorga hasta un $10 \%$ de valor a la dimensión liderazgo, evaluando la medida en que la conducta y actuaciones del equipo ejecutivo y todos los demás directivos inspira, apoya e impulsa una cultura de Calidad Total. De hecho, el estudio de casos de compañías tales como la Asahi Breweries en Japón, la Xerox en EEUU, la Dunlop en Malasia o la Dow-Corning 
en Australia, todas ellas involucradas en procesos de implantación de GCT, vienen a confirmar la relevancia del liderazgo para llevarlo a cabo con éxito (Fah, 1988; Nakajo y Kono, 1989; Kenedy, 1989; Chapman, Clarke \& Sloan, 1991).

Pero, ¿qué funciones en materia de recursos humanos son prioritarias para el directivo que persigue una gestión de excelencia?

\section{Los Retos De la Dirección}

En primer lugar, la dirección se enfrenta con el reto de llegar a concebir a las personas como los seres humanos que son, como fines en sí mismos măs que como meros medios para alcanzar los fines. "El Control Total de Calidad empieza con el factor humano y, solamente cuando éste está correctamente. situado, deben ser considerados los otros bloques" (Masaaki, 1990 pág 13).

Ciertamente, para las empresas que desean gestionar con calidad, es cada vez más evidente la necesidad de concebir a sus trabajores en su dimensión humana, creando condiciones para que se desarrollen profesional y personalmente. "La calidad total necesita de personas en toda su plenitud, y su concepción y funcionamiento no se puede realizar sin la aportación plena, voluntaria y motivada de todas las personas componentes de la empresa" (Blanco, 1999 pág 11). Así, la gestión de los recursos humanos empieza a ser reconocida como una de las principales tareas de los directivos de nuestra década. Cinco son las áreas que identifica Oakland et al (1998) entre las actividades desarrolladas por empresas modelo en gestión de calidad en relación con su capital humano. Se trata de la comunicación, la participación y el compromiso de los trabajadores, el empowerment, el entrenamiento y desarrollo y el trabajo en equipo.

La GCT no puede sobrevivir si no se articula un sistema de comunicación fluido, de doble vía e integrador. Este sistema, se debe fomentar en la práctica desde la cúspide hacia abajo. De hecho, la mayor parte de los programas empiezan con la formación de la alta dirección en la filosofía de la Calidad pero son seguidos por la definición de la visión de calidad de la organización y la comunicación de esta visión a todos los implicados (Hackman y Wageman, 1995 pág 316).

La utilización de forma efectiva de una herramienta tan sofisticada como es la comunicación, no redunda sino en beneficios para una organización. Las razones son demasiado obvias aunque no por ello su uso sea ejemplar: facilita la coordinación, posibilita el conocimiento, la formación y la participación, favorece el compromiso, la implicación y, como consecuencia, la mejora de aquello que se detecta que puede ser mejorado. Pero sobre todo, cuando la dirección ejercita una comunicación efectiva con los miembros de la organiza: ción muestra consideración, respeto y confianza hacia sus colaboradores y eso: 
tiene compensaciones. Cuando las personas son tratadas como tales, se están poniendo las bases para que respondan en consecuencia.

La formación del personal es el pilar sobre el que se aposenta la posibilidad de intervenir para el desarrollo personal y profesional: los fundamentos de la mejora continua. De acuerdo con Hackman y Wageman (1995 pág 315), la segunda práctica de GCT más usada por las empresas es la formación. Las organizaciones que desarrollan un programa de Calidad realizan altas inversiones en entrenamiento y capacitación formal. Desde un 92 por ciento de las empresas en los sectores industriales, a un 75 por ciento de las empresas de servicios que implantan la GCT, adoptan acciones formativas como parte de su programa para el cambio. Además, la mayor parte de los empleados de compañías involucradas en la GCT reciben una importante cantidad de formación anual, llegándose en los mejores casos hasta un total de 40 a 80 horas de formación al año con un presupuesto de entre 3 al 5 por ciento de la masa salarial (Eastón, 1993 pág 40). Por ejemplo, la empresa Trident, ganadora del Malcolm Baldrige del año 1996, invierte una media de 4.4 por ciento de su masa salarial en formación con objeto de diversificar las habilidades de sus trabajadores (Bemowski, 1997)

Aquellas empresas que reconocen el valor que aportan sus miembros a través de la participación, obtienen mejores resultados. El esfuerzo compartido en grupos de trabajo posibilita una visión multidisciplinar y variada que contribuye a lograr de forma más coherente la satisfacción de las necesidades de los clientes y mejora los resultados de la empresa (Milliken, 1996). A los grupos se les atribuye ventajas tales como la mejora de la satisfacción en el trabajo, la mejora de la productividad y de la calidad de los productos o servicios (Gryna, 1981; Ross y Ross, 1982). Frente a los conocidos Círculos de Calidad en Japón, la formación de grupos temporales para la solución de problemas con el objetivo de simplificar el trabajo, es la fórmula más frecuente en occidente. Aproximadamente todas las empresas en el sector industrial y en torno al 90 por ciento del sector servicios, inmersas en un programa de GCT, constituyen estos grupos en los Estados Unidos (Hackman y Wageman, 1995 pág 315).

El Presidente del comité organizador del XI Congreso de Grupos de Participación, Francisco Domínguez destaca, entre las ventajas que la participación aporta a las empresas, la humanización que conlleva reportándoles a sus miembros una mayor satisfacción en el trabajo, la consecución de una más plena realización como personas y como profesionales y la existencia de mejores relaciones personales (1999, pág 22).

Pero además del impacto positivo que la participación pueda tener en la motivación en el trabajo (Hackman y Wageman, 1995 pág 325) y en la satisfacción (Hackman y Oldham, 1976), las sugerencias de los trabajadores tienen un 
impacto económico en la @rganización (Masaaki, 1989). En Toyota el 95\% de las sugerencias realizadas por los empleados llegan a implantarse lo que viene a representar una media de en torno a las 46 ideas por empleado al año frente al $0.16 \%$ de media anual de las empresas estadounidenses. En entornos cada vez más competitivos, y en la medida en que las organizaciones reducen niveles en su jerarquía haciéndose más pequeñas, los trabajadores capacitados para tomar decisiones son más necesarios (Rao y otros, 1996 pág 464). Esta necesidad se muestra con mayor evidencia en pequeñas organizaciones donde el trabajo realizado se convierte rápidamente en ingresos.

En definitiva, los directivos deben velar por conseguir el compromiso y la participación de los miembros de la empresa para una mejor integración entre los objetivos organizacionales y las necesidades personales. "la participación demanda no inicamente el compromiso de los directivos senior en cada iniciativa de calidad, sino una involucración activa de éstos en cada grupo de trabajo vinculado a un proyecto de mejora de la calidad, con autonomía sobre el diseño y rediseño del proceso, teniendo la confianza y siendo motivados intrinsecamente y con una percepción de si mismos de que comparte la visión de calidad de la organización, la misión y los valores de la estrategia". (Morris y Haigh 1996 pág 329)

Muy relacionado con la participación está el "empowerment", la delegación o el otorgamiento de poder a los trabajadores que implica ofrecer confianza a las personas, pero, además, representa otro reto para los directivos cuyo papel sería fundamentalmente dejar trabajar a los miembros de su equipo no interfiriendo en su actividad sino facilitándoles su labor. Se trata de un paso más allá de la participación, que como consecuencia requiere que exista un equilibrio. elevado de las otras funciones: la formación, el desarrollo personal y profesional de los miembros de la empresa, la asunción de compromiso y participación en la mejora, la colaboración con los demás y un sistema transparente de comunicación.

Pero todas estas áreas de trabajo se irán materializando en la medida en que existan líderes que ostenten no sólo conocimientos, sino también una serie de habilidades personales, conceptuales y de criterio que le posibiliten poner las bases para que germine el compromiso y la satisfacción. ¿Pero dónde está el "centro de gravedad" del lider?

\section{El Ser Humano Que Hay Detrás Del lider De Calidad}

La primera cualidad indispensable de un líder es una visión, una idea clara de lo que quiere hacer (Bennis, 1994 pág 241) por lo que el líder desde el punto de vista: de la calidad deberá partir de esta premisa integrando en sus aspiraciones las pro:- 
pias de calidad. Estas últimas las resumiriamos en dos, a la hecha explícita por Deming de desarrollar un propósito de constancia para la mejora de productos y servicios, sumaríamos la de poner las bases para generar satisfacción a las personas implicadas en el proceso: clientes, trabajadores, accionistas, la sociedad en general $y$, sin duda, a uno mismo.

Las bien fundamentadas aportaciones del profesor Bennis pueden servirnos de guía para ilustrar las características del líder en este entorno. Dice el autor que es preciso escuchar la vocación propia y ser expresivo para llegar a ser líder, asentándose éste sobre tres pilares básicos: la visión, la pasión y la integridad (1995). Se trata de tener capacidad para crear una visión de referencia hacia la que avanzar, lo que provee a las personas de un puente de conexión con el futuro; capacidad de comunicación y energía para transmitir y hacer comprender esa visión e integridad. El carácter, entendido éste desde el punto de vista de la integridad, es uno de los criterios de evaluación de ejecutivos indispensable en la formación del líder pero que resultan más difíciles de enseñar y transmitir. Se trata de "el lubricante que hace posible que la organización funcione" (1997 pág 61). Con ello se genera crediblidad y confianza entre sus seguidores; sin duda, componentes esenciales para crear compromiso.

Pero más aún, en un entorno donde lo más estable es el continuo cambio y la incertidumbre sobre el futuro, el lider cultiva la agilidad física y mental que le ayuda a virar con prestancia y buen criterio de oportunidad teniendo a su disposición, como es habitual en este entorno, datos incompletos para tomar decisiones.

Estas competencias se desarrollan a partir del conocimiento que el líder tiene de sí mismo, de su capacidad para conocer sus fortalezas y debilidades con las que crear opotunidades de mejora en el plano profesional y también en el personal. El líder es un ser que no resulta ajeno al contexto con el que convive, sino que, muy al contrario, mantiene un diálogo coherente con él. Mejora y contribuye a su mejora de forma realista y mantenida de manera que su comportamiento facilita la formación de una cultura afín, dejando huella más allá de su permanencia en la empresa.

De esta forma, el sentido de la realidad que prevalece en el líder unido al deseo de logro encuentran caminos alternativos en lugar de convertirse en freno. La frase, recogida en la obra de Bennis, de un líder como el jugador de hockey Gretsky ilustra esta característica "se pierden el $100 \%$ de los tiros que no se ban intentado".

Además, nuestro autor reitera que el lider se centra en "bacer las cosas correctas" en contraposición al principio más propio de gestores de "bacer bien las cosas". Estas son cualidades altamente apreciadas pero que cada una conduce a fines diferentes. La gestión busca la eficiencia en el ascenso por la escalera del éxito; el liderazgo determina si la escalera está o no apoyada en el lugar correcto (Covey, 1995 pág. 127)

Después de lo anterior parece pertinente aclarar que el perfil del líder no es el de un ser que todo lo puede y lo sabe. Más bien es el de un facilitador que posibilita 
que las cosas ocurran en la dirección prevista. No se trata de regresar a las teorías de rasgos del liderazgo dondé se ponía el acento en los atributos recibidos a través dé la herencia, con poder de otorgar una mayor capacidad de influencia o dominio sobre otros. Esta asunción alejaría la responsabilidad de la primera persona, la posibilidad de formarse y de tomar decisiones propias para mejorar y desarrollar nuevás competencias. Por el contrario el líder no nace, no está predeterminado, se hace y; en palabras de Bennis, casi siempre se hace a sí mismo.

La GCT requiere de líderes para su implantación y además ofrece un marco idóneo para el desarrollo profesional y humano de los miembros de la organizaciớn ya sean éstos directivos o trabajadores de línea. De hecho, si nos preguntásemos acerca de las competencias requeridas por los buenos seguidores, también encon: traríamos una respuesta del profesor dado que son. básicamente, las mismas que las requeridas para ser un líder, porque “...el proceso para llegar a ser un lider es el mismo proceso que para llegar a ser un ser bumano integro" (Bennis, 1996, pág 3).

BiBI.IOGRAFIA

ANDERSEN CONSULTING \& FACONAUTO (1999) Estudio sobre la gestión de la satisfacción de los clientes en el sector automoción. Calidad, Julio-Agosto, 39-40. ARRAIZ, J.I. ;CUBEIRO, J.C.; MEDINA, J.; MORENO, J.; MULDER, E de; ORTIZ, J.M. (1999) Valor y ... jal toro! Guía práctica de la creación de valor en la empresa. Hay Group y Santillana. Madrid

BEMOWSKI. K. (1997) How did they do that? Quality Progress. March

BENNIS, W. (1994) Where leaders come from. Fortune. Septiembre, 19

BENNIS, W. (1995) Cambio y Liderazgo. Deusto

BENNIS, W. (1996) Leadership for Tomorrow. Executive Excellence, abril

BENNIS, W. (1997) In conversation with... Director, September

BLANCO RODRÍGUEZ, JM. (1999) Aspectos motivadores de la calidad en la gestión de empresas. Calidad. Abril págs 10-13.

COVEY, S.R. (1995) Los siete hábitos de la gente altamente efectiva. Paidós empresa. Barcelona.

CHAPMAN, R.L. CLARKE, P. \& SLOAN, T (1991) TQM in continuos process manufacturing: Dow-Corning (Australia) Pvt. Ltd.

International Journal of Quality and Management, Vol 8,4, 9-24.

DOMINGUEZ, F. (1999) Entrevista. Calidad. Abril, 20-22

EASTON, G.S.; (1993) The 1993 State of U.S. Total Quality Management: A BaldrigeExaminer's Perspective. California Management Review. Spring, 32-54.

FAH, C.T. (1988) A total quality control program for Dunlop Malaysia: The key issues. International Journal of Quality and Reliability Management, Vol 5, 4, 7-16. 
HACKMAN, J.R. \& OLDHAM, G.R. (1976) Motivation through the Design of Work: Test of a Theory. Organizational bebaviour and Human Performance, agosto, 250-279.

HACKMAN, J.R.; WAGEMAN, R. (1995) Total Quality Management: Empirical, conceptual and practical issues. Administrative Science Quaterly. Vol 40. 2 pp 309-342.

JOHANNSSON, C (1995) Open to suggestion. Qualit)', 2

KENNEDY, C. (1989) Xerox charts a nex strategic direction. Long Range Planning. 22 (1), $10-17$

MASAAKI, I. (1989), KAIZEN, La Clave de la Ventaja Competitiva Japonesa, México, CECSA.

MASAAKI, I (1990) Mejorando la Calidad. Ed. Gestión y Control de Calidad, S.A. Valencia.

MILLIKEN, W.F. (1996) The Eastman way, Quality Progress, Octover

MORRIS, D.S. \& HAIGH, R. H. (1996), Empowement: an endeavour to explain an enigma. Total Quality Management Vol 7, 3, 323-330.

NAKAJO, T. \& KONO, T (1989) Success through culture change in a Japanese brewery. Long Range Planning, Vol 22. 6, 29-37

OAKLAND, J.S.; OAKLAND, S (1998) The links between people management. customer satisfaction and busines resuts. Total Quality Management, vol. 9. 4-5 págs 185-190.

RAO, A.; CARR, L.P.; DAMBOLENA, I.; KOPP. R.J.; MARTIN. J.; RAFII,F.; SCHLESINGER, P.F. (1996) Total Quality Management: A Cross Functional Perspective. John Wiley \& Sons.

ROSS, J.E. \& ROSS, W.C. (1982) Japanese Qualit Circles and Productivity, Reston VA: Reston.

SERRANO, M. (1999) Modelo Siemens de Partnership, la orientación al cliente llevada a sus últimas consecuencias. Calidad, septiembre págs 14-18.

VAILL, P.; (1993) Visionary Leadership, in Allan $R$. Cohen. The Portable MBA in Management. New York, John Wiley and Sons. 